\title{
PURELY SONOCHEMICAL ROUTE FOR ORIENTED ZINC OXIDE NANOWIRE GROWTH ON ARBITRARY SUBSTRATE
}

\author{
Avinash P. Nayak ${ }^{1}$, Aaron M. Katzenmeyer ${ }^{1}$, Ja-Yeon Kim ${ }^{1}$, Min Ki Kwon ${ }^{1}$, Yasuhiro Gosho ${ }^{2}$ \\ and M. Saif Islam ${ }^{1 \dagger}$ \\ ${ }^{1}$ Electrical Engineering, University of California Davis, Davis, CA, 95616 \\ ${ }^{2}$ Yamatake Corporation and BSAC Berkley, Kanagawa, Japan \\ ${ }^{\dagger}$ Email: sislam@ucdavis.edu
}

\begin{abstract}
We report a simple sonochemical method for the seeding and synthesis of Zinc Oxide nanowire arrays that can be formed on a number of substrates that are stable in alcohol and aqueous solution. Vertically aligned ZnO NWs were synthesized from a single solution at room-ambient via ultrasonic excitation. Prior to the NW growth, a $\mathrm{ZnO}$ seed layer was deposited using the same system with a different solution. The optimal conditions to produce a high density of oriented wires along with their optical characteristics are presented for $\mathrm{ZnO}$ NWs with a significantly high growth rate compared with traditional growth techniques such as evaporation, chemical vapor deposition and sputtering. Our method promises a mass-manufacturable process for fast and inexpensive $\mathrm{ZnO}$ NW production for practical low cost electronics, photonics and energy conversion applications.
\end{abstract}

Keywords: Zinc oxide, nanowires, sonochemistry, ultrasound, solution growth.

\subsection{INTRODUCTION}

Zinc Oxide $(\mathrm{ZnO})$, a wide bandgap $(3.37 \mathrm{eV})$ semiconductor with large exciton binding energy, and high visible light transmittance, has drawn considerable interest from a variety of disciplines including engineering, material science, and the biosciences. Attractive optoelectronic properties make it a good candidate for applications in solar cells $^{[1]}$, photodetectors ${ }^{[2]}$, and displays. Its piezoelectric effect has also generated considerable interest for energy scavenging. ${ }^{[3]}$ For practical low cost nanoelectronic applications, it is important to develop mass-manufacturable processes for fast and inexpensive $\mathrm{ZnO}$ nanowire production. Vertically aligned $\mathrm{ZnO}$ nanowires (NWs) have been produced on relatively short timescales by sonication ${ }^{[4-5]}$, but this method requires costly $\mathrm{Zn}$ substrates or the deposition of $\mathrm{Zn}$ thin films to serve as a seed layer. Due to the low vapor pressure of $\mathrm{Zn}$, deposition of this material would require a dedicated deposition system, which is not practical or cost effective in many cases. Although vaporphase synthesis methods produce highly crystalline $\mathrm{ZnO} \mathrm{NWs}$, they require high temperatures which place limitations on acceptable substrates and the potential for device integration. Unlike the vapor-phase synthesis method, the hydrothermal method of growing $\mathrm{ZnO}$ NWs can be performed at much lower temperatures. The reaction time required for this synthesis of $\mathrm{ZnO} \mathrm{NWs}$ however can take several hours (or even days). In this report, we propose a method proceeding at room ambient in less time, and using only one system (750 W ultrasonic processor, Sonics and Materials). Using the method in this report, a continuous seed-layer deposition as well as an array of $\mathrm{ZnO}$ NW growth from the seed-layer is produced in a relatively short time and on various substrates over large areas. This

Energy Harvesting and Storage: Materials, Devices, and Applications, edited by Nibir K. Dhar, Priyalal S. Wijewarnasuriya, Achyut K. Dutta, Proc. of SPIE Vol. 7683, 768312 · @ 2010 SPIE CCC code: $0277-786 \mathrm{X} / 10 / \$ 18 \cdot$ doi: $10.1117 / 12.851755$ 
technique was found to be advantageous from the viewpoint of environmental impact, scalability, process time, and production cost in comparison with other techniques. ${ }^{[6-8]}$

\subsection{EXPERIMENTAL}

The seeding process and growth were performed as follows. (I) A Si $<111>$ substrate $(9-15 \Omega . \mathrm{cm})$ was immersed in a solution of isopropyl alcohol and $0.005 \mathrm{M}$ zinc acetate dihydrate $(\mathrm{C} 4 \mathrm{H} 10 \mathrm{O} 6 \mathrm{Zn}, 98+\%)$. The solution was sonicated for 15 minutes at an intensity of $19 \mathrm{~W} . \mathrm{cm}^{-2}(1.3 \mathrm{~cm}$ horn diameter) at $20 \mathrm{kHz}$. The process was repeated once to ensure a continuous layer of $\mathrm{ZnO}$. (II) An aqueous solution of $0.02 \mathrm{M}$ zinc nitrate hexahydrate $(\mathrm{Zn}(\mathrm{NO} 3) 2.6 \mathrm{H} 2 \mathrm{O}$, 99.998\%) and 0.02M hexamethylenetetramine (HMT, $(\mathrm{CH} 2) .6 \mathrm{~N} 4,99+\%)$ was stirred with a magnetic stir bar at 350 r.p.m for 10 minutes at room temperature yielding a clear solution. The solution $\mathrm{pH}$ before sonication was 7.21. (III) The Si substrate was then immersed into the aqueous solution and sonicated at $50 \%$ of the maximum amplitude of the $20 \mathrm{kHz}$ ultrasonic probe for 10 minutes providing the solution with an intensity of $21 \mathrm{~W} . \mathrm{cm}^{-2}$. The solution was refreshed two more times ( 3 cycles total). Introducing a fresh solution has been shown to improve the aspect ratio. ${ }^{[9]}$ The substrate was rinsed with DI water then dried with N2 after each cycle. (IV) The same experiment was conducted at $20 \%$ of the maximum amplitude to determine the effect of intensity providing $6 \mathrm{~W} . \mathrm{cm}^{-2}$. Samples were imaged with FEI XL30-SFEG or Hitachi S-4500 field emission Scanning Electron Microscopes. All chemical reagents used in this report were used without further purification.

The growth described above was also conducted on Au coated Si and glass substrates as a proof of concept that this method can be implemented on diverse surfaces.

\subsection{DISCUSSION AND ANALYSIS}

As seen in figure 1, a rough seed-layer is deposited on an Au coated Si substrate via the method described above. This seed-layer was found to be necessary to ensure effective growth of the arrays. The deposition of a rough seedlayer is expected to increase the density of the $\mathrm{ZnO}$ NWs in the array while having a smooth seed-layer is expected to produce more oriented $\mathrm{ZnO}$ NWs. ${ }^{[10]}$
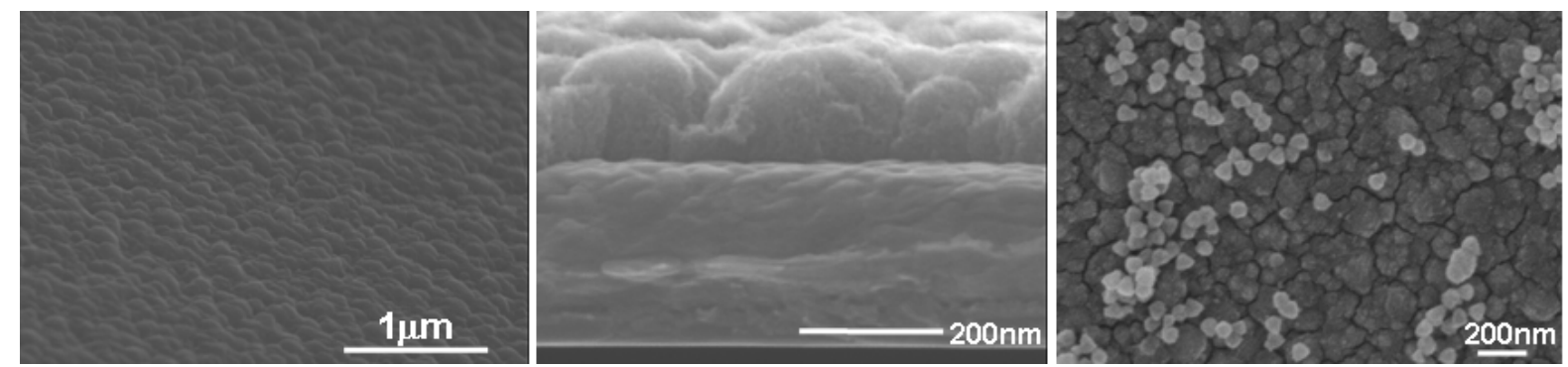

Figure 1. Seed-layer deposited on $\mathrm{Au}$ coated $\mathrm{Si}$ substrate via sonication of a $5 \mathrm{mM}$ solution of zinc acetate dihydrate in ethanol (shown in pan and cross-sectional views). A similar seed-layer can be deposited using the precursor in isopropanol (pan view). 
Experiments by Jung et. al. have shown that sonication can aid the growth of $\mathrm{ZnO}$ nanowires. ${ }^{[5]} \cdot \mathrm{OH}, \cdot \mathrm{O} 2, \cdot \mathrm{HO} 2$ and $\cdot \mathrm{H}$ radicals are produced in the sonication of water ${ }^{[5]}$ and $\cdot \mathrm{OH}$ species can promote the growth of $\mathrm{ZnO}$ nanorods through the intermediate product of zinc hyroxide. The growth of $\mathrm{ZnO} \mathrm{NW}$ arrays has been stated in the following manner ${ }^{[11-12]}$ :

$$
\begin{array}{ll}
(\mathrm{CH} 2) 6 \mathrm{~N} 4+6 \mathrm{H} 20 \rightarrow 4 \mathrm{NH} 3+6 \mathrm{HCHO} & {[1]} \\
\mathrm{NH} 3+\mathrm{H} 20 \rightarrow \mathrm{NH} 4++\mathrm{OH}- & {[2]} \\
\mathrm{Zn}(\mathrm{NO} 3) 6 \mathrm{H} 20 \rightarrow \mathrm{Zn} 2++2 \mathrm{NO} 3- & {[3]} \\
\mathrm{Zn} 2++2 \mathrm{OH}-\rightarrow \mathrm{Zn}(\mathrm{OH}) 2 & {[4]} \\
\mathrm{Zn}(\mathrm{OH}) 2 \rightarrow \mathrm{ZnO}+\mathrm{H} 2 \mathrm{O} & {[5]}
\end{array}
$$

The photoluminescence (PL) spectrum of a $\mathrm{ZnO}$ seed-layer on gold is shown in figure 2. A dominant orange peak at about $580 \mathrm{~nm}$ is seen along with a minor $\mathrm{ZnO}$ peak observed at $363 \mathrm{~nm}$. A $325 \mathrm{~nm} \mathrm{HeCd}$ laser was used to collect the spectrum.

\section{ZnO seed-layer on an Au coated Si substrate}

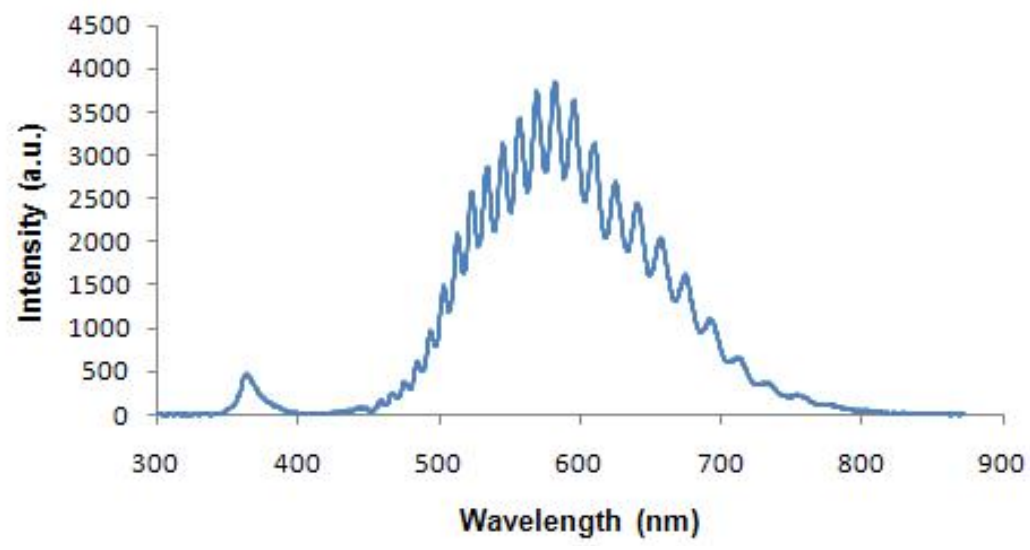

Figure 2. PL spectrum of $\mathrm{ZnO}$ thin-film seed-layer deposited via sonication. The oscillations in the orange peak result from the filter used to prevent the laser line from reaching the spectrometer.

This type of seed-layer was used to grow $\mathrm{ZnO}$ NWs under the specific conditions mentioned in the experimental section. The optimal growth conditions for oriented wires are $6 \mathrm{~W} . \mathrm{cm} 2$ intensity, $20 \%$ of the maximum amplitude, sonicated for 10 minutes each cycle. Three cycles were performed. Figure 3a shows the result. After this sample was placed on a hot-plate, hydrothermal, equimolar solution of HMT and zinc nitrate hexahydrate $(0.02 \mathrm{M})$ for two hours at $60^{\circ} \mathrm{C}$, we see that the wires get thinner but orientation stays relatively the same (figure $3 \mathrm{~b}$ ). The thin, dense wires shown in Figure $3 \mathrm{~b}$ in comparison with figure 4 shows that with larger intensity, a similar wire obtained with hotplate treatment is possible. 

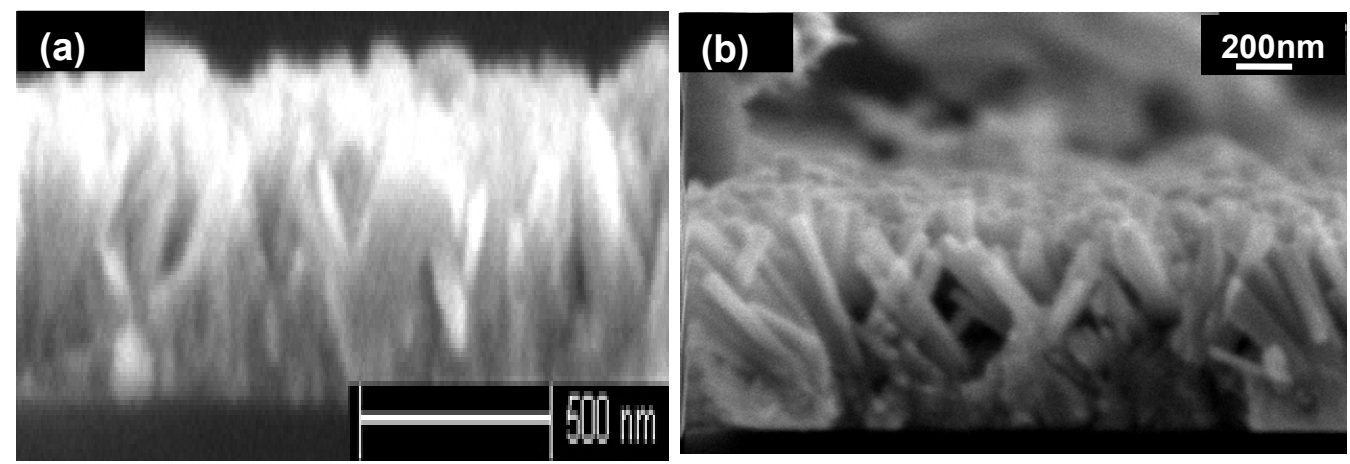

Figure 3. (a) SEM image of ZnO NWs grown with sonication at $19 \mathrm{~W} . \mathrm{cm}^{2}$ (b) Hydrothermal treatment of (a) after 2 hours at $60^{\circ} \mathrm{C}$.

This shows that oriented wires can also be grown at higher intensities $\left(21 \mathrm{~W} . \mathrm{cm}^{-2}\right)$ removing the need to introduce a hot-plate solution into the synthesis. At $50 \%$ of the maximum amplitude of the $20 \mathrm{kHz}$ ultrasonic probe oriented $\mathrm{ZnO}$ NWs can be synthesized as seen in figure 4.

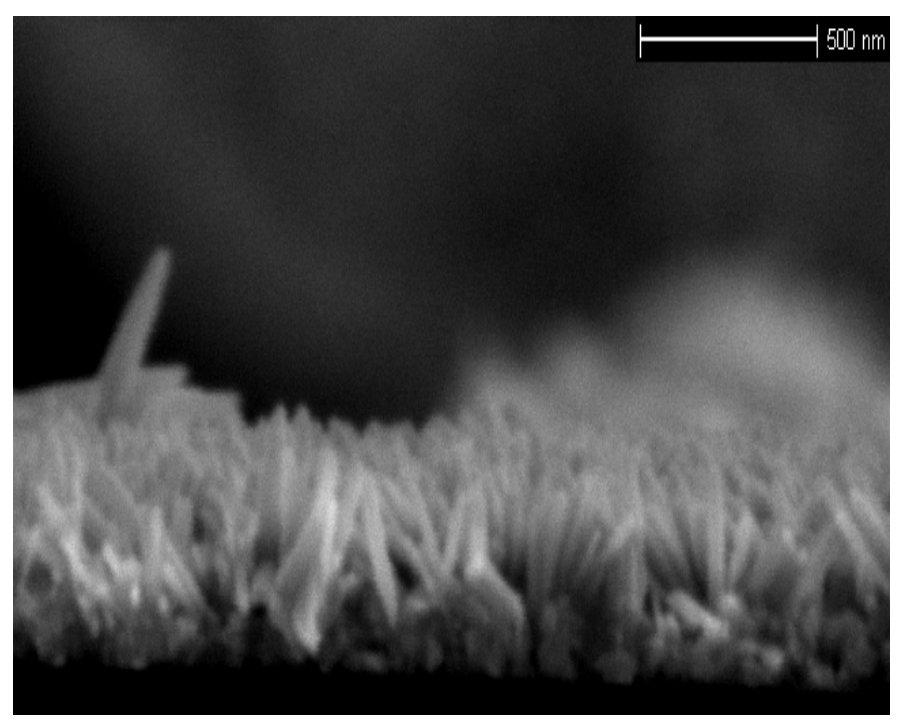

Figure 4. $\mathrm{ZnO}$ nanowires produced using an intensity of $21 \mathrm{~W} \cdot \mathrm{cm}^{-2}$.

The PL spectrum of a $\mathrm{ZnO}$ NW array under these conditions $\left(21 \mathrm{Wcm}^{-2}\right)$ is shown in figure 5 . This, in comparison to figure 2 shows a strong peak at the $\mathrm{ZnO}$ UV wavelength $(377 \mathrm{~nm})$ along with the visible light defect peak ${ }^{[13]}$. This suggests that reasonable quality $\mathrm{ZnO} \mathrm{NWs}$ were seeded by the low quality $\mathrm{ZnO}$ deposition under our mild growth process without the need for subsequent treatments such as annealing. 


\section{$\mathrm{PL}$ of $\mathrm{ZnO}$ array on an Au coated Si substrate}

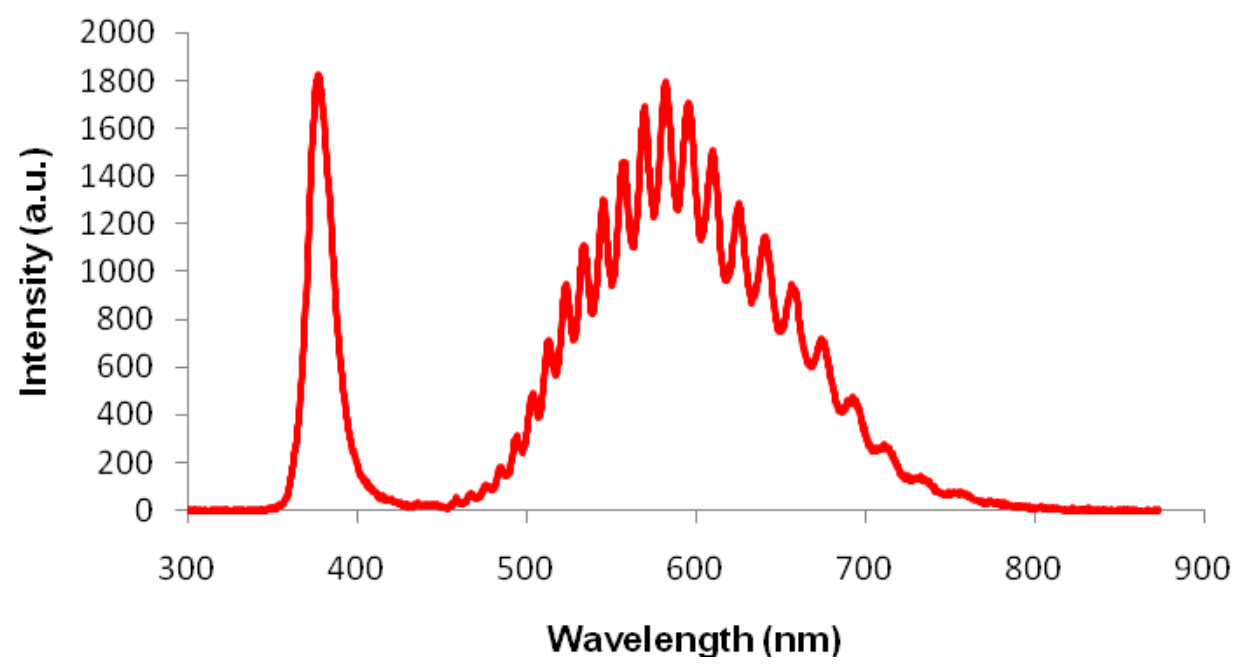

Figure 5. PL spectrum of a $\mathrm{ZnO}$ array produced via Sonication at $21 \mathrm{Wcm}^{-2}$.

Raman scattering was performed to assess the vibrational properties of the NWs. Both ZnO NW arrays investigated displayed a peak at $438 \mathrm{~cm}^{-1}$ which corresponds to the $\mathrm{E}_{2}{ }^{\text {High }}$ nonpolar phonon mode, associated with oxygen. ${ }^{[14]}$ The linewidth broadening of the peak to beyond a FWHM of $5 \mathrm{~cm}^{-1}$ has been attributed to isotopic inhomogeneity of the oxygen atoms in the material (i.e. the presence of $18 \mathrm{O}$ in addition to $16 \mathrm{O}$ ). ${ }^{[15]}$

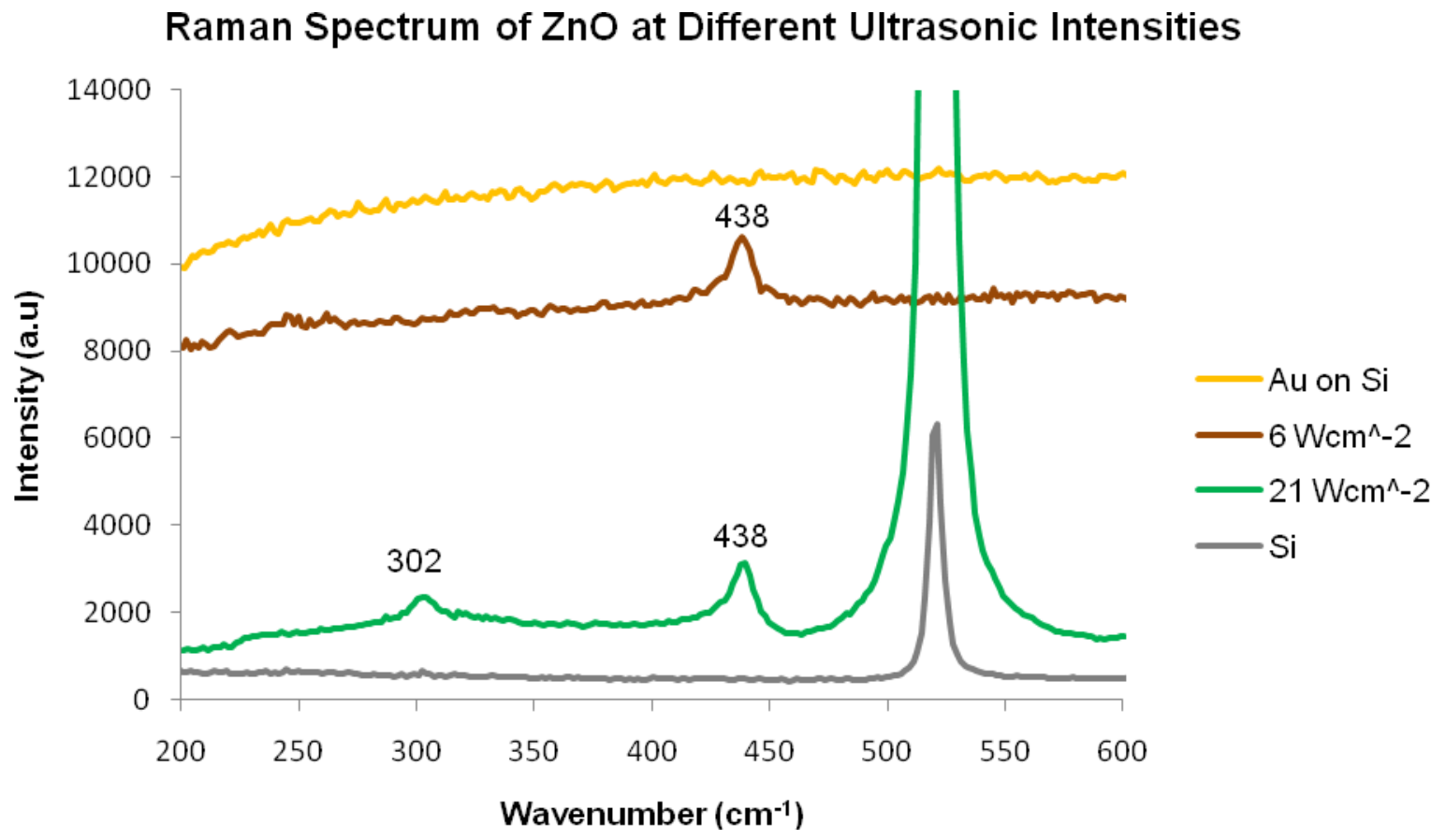

Figure 6. Raman scattering of $\mathrm{ZnO}$ samples prepared at different ultrasonic intensities. 
The $302 \mathrm{~cm}^{-1}$ peak seen wide in the $21 \mathrm{Wcm}^{-2}$ sample is attributed to the Si substrate. ${ }^{[16]}$

To show that $\mathrm{ZnO} \mathrm{NW}$ arrays can be grown on non-metallic and inexpensive surfaces, we deposited a $\mathrm{ZnO}$ seedlayer and then grew the $\mathrm{ZnO}$ nanowires on glass. Figure 7 shows the result of this growth.

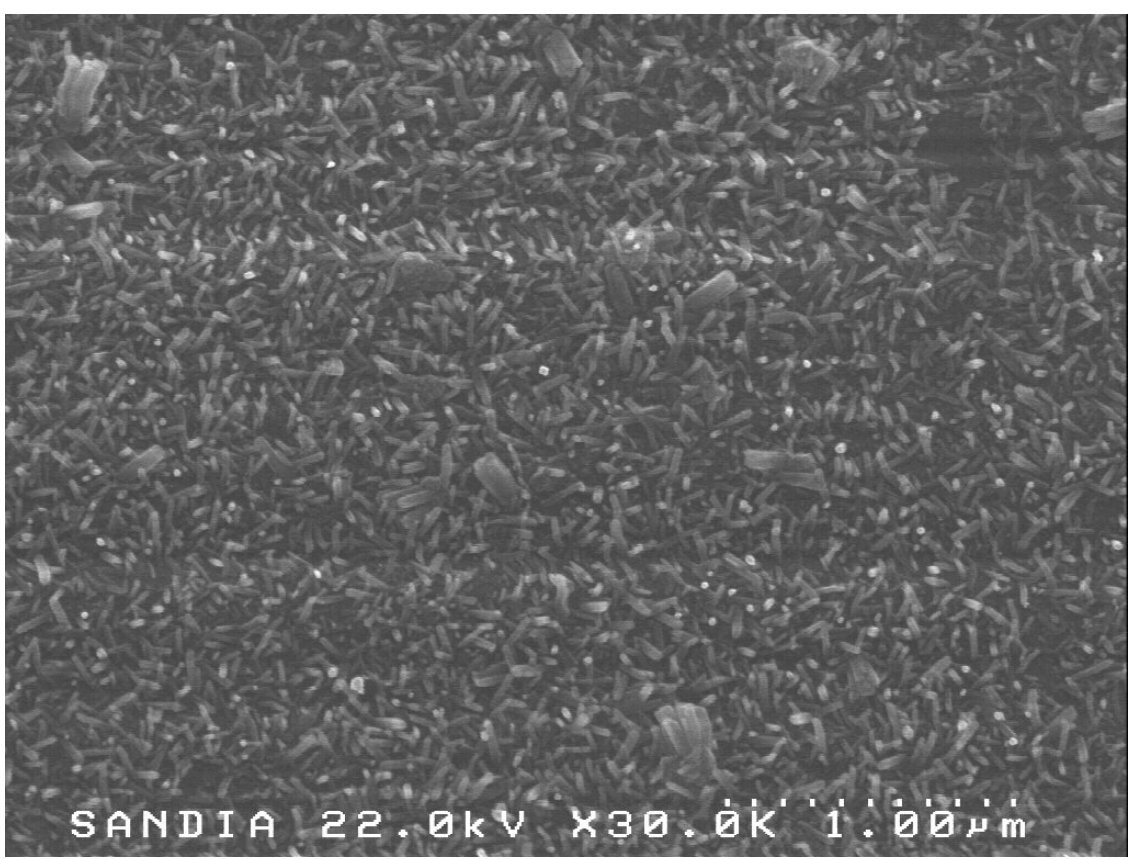

Figure 7. $\mathrm{ZnO}$ nanowires grown on glass.

Our average growth rate is $\sim 600 \mathrm{nmh}^{-1}$. Compared with other room-ambient approaches, our growth time is significantly smaller than other reported sonication syntheses ${ }^{[5]}$.Compared to other literature, our growth rate is $>10$ times that of conventional hydrothermal methods used which have a growth rate of $40 \mathrm{nmh}^{-1}$. ${ }^{[17]}$

In this paper, we have presented an economical and rapid approach for growing $\mathrm{ZnO} \mathrm{NW}$ arrays. Using just one system, we were able to deposit and grow the wires on conducting and insulating substrates of varied quality by sonochemical means. It is expected that this method will be useful in fabricating stand-alone and also integrated optoelectronic devices. 


\section{REFERENCES}

[1] L. E. Greene, Et Al.," Low-Temperature Wafer-Scale Production Of ZnO Nanowire Arrays. " Angew. Chem. Int. Ed. 42, 3031-3034 (2003).

[2] O. Lupan, Et Al., "Focused-Ion-Beam Fabrication Of Zno Nanorod-Based UV Photodetector Using The InSitu Lift-Out Technique" Physica Status Solidi 11, 2673-2678 (2008).

[3] Y. Qin, X. Wang, And Z.L. Wang, "Microfibre-Nanowire Hybrid Structure For Energy Scavenging." Nature Letters 451, 809-813 (2008)

[4] X. Hu, Y.Z., S. Wang, "Sonochemical And Microwave-Assisted Synthesis Of Linked Single-Crystalline ZnO Rods" Materials Chemistry And Physics 88, 421-426 (2004).

[5] S. Jung, Et Al.,"A Sonochemical Method For Fabricating Aligned ZnO Nanorods" Advanced Materials 19(5),749-753 ( 2007).

[6] D. Banerjee, Et Al., "Large-Quantity Free-Standing ZnO Nanowires" Applied Physics Letters, 83(10), 2061-2063 (2003).

[7] M.J. Zheng, Et Al., "Fabrication And Optical Properties Of Large-Scale Uniform Zinc Oxide Nanowire Arrays By One-Step Electrochemical Deposition Technique" Chemical Physics Letters, 123-128 (2008).

[8] P. X. Gao, Y. Ding, And Z.L. Wang, "Crystallographic Orientation-Aligned ZnO Nanorods Grown By A Tin Catalyst" Nano Letters 3(9), 1315-1320 (2003)

[9] J.B. Baxter, Et Al., "Synthesis And Characterization Of ZnO Nanowires And Their Integration Into DyeSensitized Solar Cells" Nanotechnology 17,304-312 (2006).

[10] S Xu, Et Al., "Density-Controlled Growth Of Aligned ZnO Nanowire Arrays By Seedless Chemical Approach On Smooth Surfaces" Materials Research Society 23(8), 2072-2077 (2008).

[11] M. A. Beckett, I. Hua, "Impact Of Ultrasonic Frequency On Aqueous Sonoluminescence And Sonochemistry." Physical Chemistry. A105, 3796-3802 (2001).

[12] M. Breedon, Et Al., "Zno Nanostructured Arrays Grown From Aqueous Solutions On Different Substrates" Iconn, 9-12 (2008). 
[13] V.A. Fonoberov, K. A. Alim, And A.A. Balandin, "Photoluminescence Investigation Of The Carrier Recombination Processes In Zno Quantum Dots And Nanocrystals" Physical Review B 73, 1-9 (2006).

[14] Khan A. Alim, Et Al., "Micro-Raman Investigation Of Optical Phonons In ZnO Nanocrystals. " Journal Of Applied Physics 97(124313), 1-5 (2005).

[15] J. Serrano, Et Al.," Dispersive Phonon Linewidths: The E2 Phonons Of ZnO" Phys Rev Lett, 90(5), 1-4 (2003).

[16] S. Hayashi, K. Yamamoto, "Optical Properties Of Si-Rich SiOz Films In Relation With Embedded Si Mesoscopic Particles." Journal Of Luminescence 70, 352-363 (1996).

[17] H. Yu, Et Al., "A General Low-Temperature Route For Large-Scale Fabrication Of Highly Oriented ZnO Nanorod/Nanotube Arrays" Jacs Communications 127(8), 2378-2379 (2005). 